\title{
Augmented MVDR Spectrum-Based Frequency Estimation for Unbalanced Power Systems
}

\author{
Yili Xia, Member, IEEE and Danilo P. Mandic, Fellow, IEEE
}

\begin{abstract}
A robust technique for online estimation of the fundamental frequency of both balanced and unbalanced threephase power systems is proposed. This is achieved by extending the recently introduced iterative frequency estimation method based on minimum variance distortionless response (MVDR) spectrum [1], in order to enhance its robustness in unbalanced system conditions. The approach is made optimal for the secondorder noncircular nature of the unbalanced complex-valued system voltage, by combining the iterative MVDR (I-MVDR) frequency estimation and the complete available (augmented) second-order statistics. Such an approach makes it possible to eliminate the otherwise unavoidable estimation bias in unbalanced system conditions. It is also shown that the proposed method approaches the theoretical Cramer-Rao lower bound (CRLB), which we rigorously derive for the vector parameter in power systems. Simulations over a range of unbalanced conditions, including voltage sags, the presence of higher-order harmonics, and for real-world unbalanced power systems, support the analysis.
\end{abstract}

Index Terms-Complex noncircularity, frequency estimation, minimum variance distortionless response (MVDR) spectrum, unbalanced power systems, pseudocovariance.

\section{INTRODUCTION}

$\mathbf{F}$ REQUENCY is an important power quality parameter that is only allowed to vary within a small predefined range, a consequence of the dynamic unbalance between the generation and the load [2]. Its accurate estimation is essential, as maintaining the nominal frequency value is a prerequisite for both the stability of the grid and for normal operation of electrical devices [3]. Frequency is also a key parameter in the control of distributed grids, where it can be used to determine the harmonic contents of currents drawn by nonlinear loads.

To deal with these issues, research into fast and accurate frequency estimation has attracted much attention. Architecture and algorithms for this purpose include zero crossing techniques [4], phase-locked loops (PLL) [5], least-squares based adaptive filters [6], recursive Newton-type estimation

Manuscript received July 23, 2012; revised December 17, 2012; accepted December 18, 2012. Date of publication May 14, 2013; date of current version June 5, 2013. The Associate Editor coordinating the review process was D. Petri.

Y. Xia is with the School of Information Science and Engineering, Southeast University, Nanjing 210096, China, and also with the Department of Electrical and Electronic Engineering, Imperial College London, London SW7 2AZ, U.K. (e-mail: yili.xia06@gmail.com).

D. Mandic is with the Department of Electrical and Electronic Engineering, Imperial College London, London SW7 2AZ, U.K. (e-mail: d.mandic@imperial.ac.uk).

Color versions of one or more of the figures in this paper are available online at http://ieeexplore.ieee.org.

Digital Object Identifier 10.1109/TIM.2013.2253160
[7], extended complex Kalman filters (ECKF) [8], adaptive notch filters [9] and demodulation methods [10]. Popular solutions also include eigenvalue methods, such as multiple signal classification (MUSIC) [11] and estimation via rotational invariance (ESPRIT) techniques [12]; however, their computational burden limits their use in practical applications.

According to the International Standard on the measurement of power quality parameters, IEC 61000-4-30, the frequency in multiphase $50 / 60 \mathrm{~Hz}$ ac power supply systems is measured from a single reference channel [13]. To characterize power system frequency from multiphase measurements, while enhancing robustness, it is convenient to exploit the complexvalued system voltage, obtained from the Clarke's $\alpha \beta$ transformation [10]; this way all available information among the three-phase reference channels is used, at a cost of increasing computational complexity [6], [8], [10].

Most frequency estimation algorithms in the complex domain $\mathbb{C}$ work well under normal balanced power system conditions. There also exist algorithms that operate well in noisy environments and in the presence of frequency deviation, however, vast majority suffer from performance degradation under unbalanced voltage conditions. These occur when different amplitudes within the three-phase voltage (voltage sag) in one or two phases is taking place, resulting in the negative sequence and an unavoidable estimation error, represented by oscillations at double the system frequency. One solution is to employ the symmetrical component transformation to extract the positive sequence, so that the standard phase angle extraction algorithms designed for frequency estimation in balanced power systems can be directly applied [14], [15]. We have recently proposed a scheme that exploits the noncircular statistical nature of the complex-valued system voltage under the unbalanced power systems to extend frequency estimation algorithms into a more general form, which caters for the system imbalance [16], [17]. It was shown that under unbalanced conditions, the Clarke's transformed system voltage is second-order noncircular (improper), for which the probability density function is not rotation invariant [18]. For such a noncircular voltage $\mathbf{v}$, the powers in the real and imaginary part are different; in this case, the covariance matrix $\mathrm{C}=E\left[\mathbf{v v}^{H}\right]$ does not contain all the necessary information, and the pseudocovariance matrix $\mathrm{P}=E\left[\mathbf{v v}^{T}\right]$ should also be considered to describe complete second-order statistical behavior [19]-[21].

Recently, an accurateand fast frequency estimation algorithm has been proposed in [1], based on the minimum variance distortionless response (MVDR) spectrum that generates 
sharp peaks in the spectrum, where frequency components are located [22], [23]. This algorithm employs a gradient descent-based least mean square (LMS) adaptive scheme to iteratively estimate the fundamental system frequency. By maximizing the MVDR spectrum, a superior performance of this scheme over the ECKF and the demodulation algorithms was verified. However, despite its efficiency and an elegant analysis framework, iterative MVDR (I-MVDR) frequency estimation can operate only in balanced system conditions.

In this paper, we extend the frequency estimation method based on the MVDR spectrum so as to make it possible to operate with enhanced accuracy, robustness, and no bias, under unbalanced system conditions. To deal with the noncircularity of unbalanced voltages, the proposed augmented I-MVDR (AI-MVDR) algorithm incorporates the pseudocovariance matrix into its iterative frequency identification, thus offering more degrees of freedom in estimation, and a unifying estimator for both balanced and unbalanced conditions. We first prove that for unbalanced power systems the original I-MVDR algorithm renders biased frequency estimates, owing to its use of the strictly linear model that cannot cater for the noncircularity of the complex system voltage. To solve this problem, compensation terms are introduced based on the consideration of both the covariance and pseudocovariance matrix, and are incorporated into the iterative gradient optimization to give unbiased and minimum variance performance. For rigor, the performance of the proposed AI-MVDR algorithm is verified against the theoretical Cramer-Rao lower bound (CRLB), and our recently introduced adaptive unbiased frequency estimator, called the augmented complex LMS (ACLMS), [16], [17], on both synthetic and real world case studies.

\section{UNBALANCED THREE-PHASE POWER SySTEMS}

The three-phase voltages of a power system in a noise-free environment can be represented in a discrete time form as

$$
\begin{aligned}
& v_{a}(k)=V_{a} \cos (\Omega k \Delta \mathrm{T}+\phi) \\
& v_{b}(k)=V_{b} \cos \left(\Omega k \Delta \mathrm{T}+\phi-\frac{2 \pi}{3}\right) \\
& v_{c}(k)=V_{c} \cos \left(\Omega k \Delta \mathrm{T}+\phi+\frac{2 \pi}{3}\right)
\end{aligned}
$$

where $V_{a}, V_{b}, V_{c}$ are the peak values of each fundamental voltage component at time instant $k, \Delta \mathrm{T}=\frac{1}{f_{s}}$ is the sampling interval, where $f_{s}$ is the sampling frequency, $\phi$ is the initial phase, and $\Omega=2 \pi f_{\mathrm{o}}$ is angular frequency of the voltage signal, with $f_{\mathrm{o}}$ being the system frequency. The three-phase voltage is routinely transformed by the orthogonal $\alpha \beta 0$ transformation matrix [10] into a zero-sequence $v_{0}$ and the direct and quadrature-axis components, $v_{\alpha}$ and $v_{\beta}$, as

$$
\left[\begin{array}{l}
v_{0}(k) \\
v_{\alpha}(k) \\
v_{\beta}(k)
\end{array}\right]=\sqrt{\frac{2}{3}}\left[\begin{array}{ccc}
\frac{\sqrt{2}}{2} & \frac{\sqrt{2}}{2} & \frac{\sqrt{2}}{2} \\
1 & -\frac{1}{2} & -\frac{1}{2} \\
0 & \frac{\sqrt{3}}{2} & -\frac{\sqrt{3}}{2}
\end{array}\right]\left[\begin{array}{l}
v_{a}(k) \\
v_{b}(k) \\
v_{c}(k)
\end{array}\right]
$$

The factor $\sqrt{2 / 3}$ ensures that the system power is invariant under this transformation. In balanced system conditions, $V_{a}, V_{b}, V_{c}$ are identical, giving $v_{0}(k)=0, v_{\alpha}(k)=$ $A \cos (\Omega k \Delta \mathrm{T}+\phi)$ and $v_{\beta}(k)=A \sin (\Omega k \Delta \mathrm{T}+\phi)$. In practice, normally, only the $v_{\alpha}$ and $v_{\beta}$ parts are used to form the complex system voltage $v(k)$, given by [10]

$$
v(k)=v_{\alpha}(k)+j v_{\beta}(k)=A e^{j(\Omega k \Delta \mathrm{T}+\phi)}=A e^{j\left(\omega_{0} k+\phi\right)}
$$

where $\omega_{\mathrm{o}}=\Omega \Delta \mathrm{T}$ is the normalised angular frequency, and $v_{\alpha}(k)$ and $v_{\beta}(k)$ represent the orthogonal coordinates of a point whose position is time variant at a rate proportional to the system frequency. For a constant $f_{s}$, the probability density function of $v(k)$ is rotation invariant, since both $v$ and $v e^{j \theta}$ have the same distribution for any real $\theta$. This means that $v(k)$ is second order circular (proper) and with equal powers in $v_{\alpha}$ and $v_{\beta}$, thus the covariance matrix $\mathrm{C}$ can fully describe the second order statistics [18] while the pseudocovariance matrix $\mathrm{P}=E\left[\mathbf{v v} \mathbf{v}^{T}\right]=\mathbf{0}$ vanishes. However, when the threephase power system deviates from its nominal condition, such as under different levels of dips or transients, $V_{a}, V_{b}, V_{c}$ are not identical, and the complex voltage from (3) becomes [16]

$$
v(k)=A e^{j\left(\omega_{0} k+\phi\right)}+B e^{-j\left(\omega_{0} k+\phi\right)}
$$

where

$$
\begin{aligned}
& A=\frac{\sqrt{6}\left(V_{a}+V_{b}+V_{c}\right)}{6} \\
& B=\frac{\sqrt{6}\left(2 V_{a}-V_{b}-V_{c}\right)}{12}-j \frac{\sqrt{2}\left(V_{b}-V_{c}\right)}{4} .
\end{aligned}
$$

This expression is theoretically accurate for both the balanced and unbalanced conditions, and should be used in time-varying environments. For balanced system conditions, $V_{a}=V_{b}=V_{c}$ and $B=0$, whereas for unbalanced conditions, $B \neq 0$, causing the samples of $v(k)$ generated from (4) to deviate from the circle with constant radius, making the distribution of $v(k)$ rotation dependent (noncircular). This, in turn, produces nonzero values of the pseudocovariance matrix, while the covariance matrix $\mathrm{C}$ does not have sufficient degrees of freedom to describe full second-order statistics. Hence, current, strictly linear frequency trackers in $\mathbb{C}$, which are based solely on the covariance matrix are suboptimal for unbalanced systems, and the pseudocovariance matrix should also be considered to use all the available statistics [20].

\section{THE AUgMENTED ITERATIVE MVDR FREQUENCY ESTIMATION FOR UNBALANCED POWER SYSTEMS}

\section{A. Iterative MVDR (I-MVDR) Algorithm}

The MVDR spectrum is designed to exhibit sharp peaks at the location of desired frequency components, defined as

$$
S_{\operatorname{MVDR}}(\omega)=\frac{1}{\mathbf{s}^{H}(\omega) \mathrm{C}^{-1} \mathbf{s}(\omega)}
$$

where $\mathrm{C}$ is the covariance matrix of the complex system voltage $v(k)$, while the vector of complex exponentials

$$
\mathbf{s}(\omega)=\left[1, e^{-\jmath \omega}, \ldots, e^{-\jmath(K-1) \omega}\right]^{T}
$$

is also termed the frequency scanning vector, and $K$ is the dimension of the square covariance matrix $C$. When the fundamental component $\omega$ of the frequency scanning vector is the same as one of the frequency components that are contained 
in the input signal (and reflected in the covariance matrix), the MVDR estimator in (6) returns the power associated with the frequency component in hand. However, in the presence of noise, when the power of each frequency component is larger than the noise power, the denominator of (6) has several local minima for every angular frequency $\omega$. Physically, each local minimum is associated with the angular frequency of one of the frequency components present, and the local minimum that has the smallest absolute value indicates the fundamental angular frequency. When only the fundamental frequency component is present and contaminated with additive white noise, the dimension of the covariance matrix $\mathrm{C}$ is set to $K=2$; this is sufficient for frequency estimation when only the fundamental frequency is considered [22], [23].

The I-MVDR in [1] is based on minimizing the cost function

$$
J(k)=\mathbf{s}^{H}(\omega(k)) \mathbf{C}^{-1} \mathbf{s}(\omega(k))
$$

using a LMS stochastic gradient [24],

$$
\omega(k+1)=\omega(k)-\mu \nabla_{\omega} J(k)
$$

where $\omega(k)$ denotes the estimated angular frequency at time instant $k$, and $\mu$ is the step-size which controls the tradeoff between convergence speed and the estimation accuracy. For convenience, denote the inverse of the covariance matrix by ${ }^{1}$

$$
\mathrm{C}^{-1}=\left[\begin{array}{ll}
i c_{11} & i c_{12} \\
i c_{21} & i c_{22}
\end{array}\right]
$$

In this way, the cost function can be further simplified as

$$
\begin{aligned}
J(k) & =\left[\begin{array}{ll}
1 & e^{J \omega(k)}
\end{array}\right]\left[\begin{array}{ll}
i c_{11} & i c_{12} \\
i c_{21} & i c_{22}
\end{array}\right]\left[\begin{array}{c}
1 \\
e^{-j \omega(k)}
\end{array}\right] \\
& =i c_{11}+i c_{12} e^{-j \omega(k)}+i c_{21} e^{\jmath \omega(k)}+i c_{22}
\end{aligned}
$$

The covariance matrix is Hermitian, $i c_{21}=i c_{12}^{*}$, thus

$$
\begin{aligned}
\nabla_{\omega} J(k) & =\frac{\partial J(k)}{\partial \omega(k)} \\
& =-j i c_{12} e^{-\jmath \omega(k)}+j i c_{12}^{*} e^{\jmath \omega(k)} \\
& =-J\left(\Re\left(i c_{12}\right)+J \Im\left(i c_{12}\right)\right)(\cos (\omega(k))-\jmath \sin (\omega(k))) \\
& +J\left(\Re\left(i c_{12}\right)-J \Im\left(i c_{12}\right)\right)(\cos (\omega(k))+\jmath \sin (\omega(k))) \\
& =-2\left(\sin (\omega(k)) \Re\left(i c_{12}\right)-\cos (\omega(k)) \Im\left(i c_{12}\right)\right)
\end{aligned}
$$

where $\Re(\cdot)$ and $\Im(\cdot)$ denote the real and imaginary part operators. The I-MVDR algorithm is then given by ${ }^{2}$

$$
\omega(k+1)=\omega(k)+\mu\left(\sin (\omega(k)) \Re\left(i c_{12}\right)-\cos (\omega(k)) \Im\left(i c_{12}\right)\right)
$$

\section{B. Suboptimality of I-MVDR for Unbalanced Systems}

In the steady state, after the algorithm in (13) converges, the gradient $\nabla_{\omega(k)} J(k)$ has values close to zero, so that

$$
\sin (\omega(k)) \Re\left(i c_{12}\right)-\cos (\omega(k)) \Im\left(i c_{12}\right) \approx 0
$$

which can be rewritten as

$$
\tan (\omega(k))=\frac{\sin (\omega(k))}{\cos (\omega(k))}=\frac{\Im\left(i c_{12}\right)}{\Re\left(i c_{12}\right)}
$$

\footnotetext{
${ }^{1}$ The elements of the inverse covariance matrix are denoted by "i", for instance $i c_{11}$ is the first element of the inverse covariance matrix.

${ }^{2}$ The factor 2 in (12) is absorbed into the step-size $\mu$ in (13).
}

and thus the estimated frequency

$$
\omega(k)=\tan ^{-1}\left(\frac{\Im\left(i c_{12}\right)}{\Re\left(i c_{12}\right)}\right)
$$

Consider again the $2 \times 2$ covariance matrix of $v(k)$, given by

$$
\mathrm{C}=\left[\begin{array}{cc}
E\left[v(k) v^{*}(k)\right] & E\left[v(k) v^{*}(k-1)\right] \\
E\left[v(k-1) v^{*}(k)\right] & E\left[v(k-1) v^{*}(k-1)\right]
\end{array}\right]
$$

For unbalanced systems, when $v(k)$ is given in (4), and

$$
\begin{aligned}
E\left[v(k) v^{*}(k)\right] & =E\left[\left(A A^{j\left(\omega_{0} k+\phi\right)}+B e^{-j\left(\omega_{0} k+\phi\right)}\right)\left(A e^{-j\left(\omega_{0} k+\phi\right)}+B^{*} e^{j\left(\omega_{0} k+\phi\right)}\right)\right] \\
& =E\left[|A|^{2}+A B e^{-2 J\left(\omega_{0} k+\phi\right)}+A B^{*} e^{2\left(\omega_{0} k+\phi\right)}+|B|^{2}\right] \\
& =|A|^{2}+|B|^{2}
\end{aligned}
$$

and similarly

$$
\begin{aligned}
E\left[v(k) v^{*}(k-1)\right] & =|A|^{2} e^{\jmath \omega_{0}}+|B|^{2} e^{-\jmath \omega_{0}} \\
E\left[v(k-1) v^{*}(k)\right] & =|A|^{2} e^{-\jmath \omega_{0}}+|B|^{2} e^{j \omega_{0}} \\
E\left[v(k-1) v^{*}(k-1)\right] & =|A|^{2}+|B|^{2}
\end{aligned}
$$

to give

$$
\mathrm{C}=\left[\begin{array}{cc}
|A|^{2}+|B|^{2} & |A|^{2} e^{\jmath \omega_{0}}+|B|^{2} e^{-\jmath \omega_{\mathrm{o}}} \\
|A|^{2} e^{-\jmath \omega_{\mathrm{o}}}+|B|^{2} e^{\jmath \omega_{\mathrm{o}}} & |A|^{2}+|B|^{2}
\end{array}\right]
$$

The variance of the complex voltage $\sigma_{v}^{2}$ is $c_{11}=\sigma_{v}^{2}=|A|^{2}+$ $|B|^{2}$, while

$$
\begin{aligned}
C^{-1} & =\frac{\left[\begin{array}{cc}
|A|^{2}+|B|^{2} & -\left(|A|^{2} e^{2 \omega_{0}}+|B|^{2} e^{-j \omega_{0}}\right) \\
-\left(|A|^{2} e^{-J \omega_{0}}+|B|^{2} e^{j \omega_{0}}\right) & |A|^{2}+|B|^{2}
\end{array}\right]}{\left(|A|^{2}+|B|^{2}\right)^{2}-\left(|A|^{2} e^{\jmath \omega_{0}}+|B|^{2} e^{-J \omega_{0}}\right)\left(|A|^{2} e^{-j \omega_{0}}+|B|^{2} e^{\jmath \omega_{0}}\right)} \\
= & \frac{\left[\begin{array}{cc}
|A|^{2}+|B|^{2} & -\left(|A|^{2} e^{j \omega_{0}}+|B|^{2} e^{-j \omega_{0}}\right) \\
-\left(|A|^{2} e^{-J \omega_{0}}+|B|^{2} e^{j \omega_{0}}\right) & |A|^{2}+|B|^{2}
\end{array}\right]}{2|A|^{2}|B|^{2}\left(1-\cos \left(2 \omega_{0}\right)\right)}
\end{aligned}
$$

, and therefore,

$$
\begin{aligned}
i c_{12} & =-\frac{|A|^{2} e^{\jmath \omega_{0}}+|B|^{2} e^{-\jmath \omega_{o}}}{2|A|^{2}|B|^{2}\left(1-\cos \left(2 \omega_{0}\right)\right)} \\
& =-\frac{\left(|A|^{2}+|B|^{2}\right) \cos \left(\omega_{0}\right)+J\left(|A|^{2}-|B|^{2}\right) \sin \left(\omega_{0}\right)}{2|A|^{2}|B|^{2}\left(1-\cos \left(2 \omega_{0}\right)\right)}
\end{aligned}
$$

From (16), the steady-state I-MVDR frequency estimator is

$$
\begin{aligned}
\omega(k) & =\tan ^{-1}\left(\frac{\Im\left(i c_{12}\right)}{\Re\left(i c_{12}\right)}\right) \\
& =\tan ^{-1}\left(\frac{|A|^{2}-|B|^{2}}{|A|^{2}+|B|^{2}} \tan \left(\omega_{0}\right)\right) \\
& =\tan ^{-1}\left(\frac{1-r}{1+r} \tan \left(\omega_{0}\right)\right)
\end{aligned}
$$

where $r=\frac{|B|^{2}}{|A|^{2}}$ is the system imbalance coefficient, a ratio between the squared envelops of the positive and negative sequences in (4). In unbalanced conditions, $B \neq 0$, and $\frac{1-r}{1+r}<1$, thus causing an unavoidable bias (under-estimation) in the estimation of the true system frequency $\omega_{0}$, when using the iterative frequency estimation scheme in (13).

\section{The Proposed AI-MVDR Algorithm}

To deal with the bias in the MVDR spectrum-based frequency estimation, we can employ the widely linear modelbased MVDR method, introduced in [25], which is optimal for both complex-valued circular and noncircular signals. However, its computational complexity is relatively high due to the matrix inverse of the augmented covariance matrix $\mathrm{C}_{a}=$ 
$\left[\mathrm{C}, \mathrm{P} \vdots \mathrm{P}^{*}, \mathrm{C}^{*}\right]$, which is four times the size of the standard covariance matrix. We here introduce a more computationally efficient method to derive the unbiased frequency estimator, by considering a modification of (25). Since physically, the estimation bias arises from the term $\frac{1-r}{1+r}$, a natural solution would be to use all the available statistical information to calculate this ratio, and to incorporate its inverse into the estimator in (13), so that an unbiased estimator becomes

$$
\begin{aligned}
\omega(k+1) & =\omega(k)+\mu\left(\sin (\omega(k)) \Re\left(i c_{12}\right)(1+r)\right. \\
& \left.-\cos (\omega(k)) \Im\left(i c_{12}\right)(1-r)\right)
\end{aligned}
$$

from which the system frequency can be calculated as

$$
f(k)=\frac{f_{s}}{2 \pi} \omega(k)
$$

However, this is not straightforward, as the covariance matrix in (22) does not have sufficient degrees of freedom to explain $r$, as it only provides a relationship between two unknown parameters $A$ and $B$, that is

$$
|A|^{2}+|B|^{2}=c_{11}
$$

and not the actual values of $|A|$ and $|B|$. To solve (28), we employ another relationship between $A$ and $B$, contained in the pseudocovariance matrix, defined as [19], [20]

$$
\begin{aligned}
\mathrm{P} & =\left[\begin{array}{cc}
E[v(k) v(k)] & E[v(k-1) v(k)] \\
E[v(k) v(k-1)] & E[v(k-1) v(k-1)]
\end{array}\right] \\
& =\left[\begin{array}{cc}
2 A B & 2 A B \cos \left(\omega_{0}\right) \\
2 A B \cos \left(\omega_{\mathrm{o}}\right) & 2 A B
\end{array}\right]
\end{aligned}
$$

From the first element in $\mathrm{P}, p_{11}$, we obtain $B=\frac{p_{11}}{2 A}$, and

$$
|B|^{2}=\frac{\left|p_{11}\right|^{2}}{4|A|^{2}}
$$

Substituting (30) for (28), we have

$$
4|A|^{4}-4 c_{11}|A|^{2}+\left|p_{11}\right|^{2}=0
$$

for which the two roots can be found as

$$
|A|^{2}=\frac{c_{11} \pm \sqrt{\left|c_{11}\right|^{2}-\left|p_{11}\right|^{2}}}{2}
$$

while from (28), we have

$$
|B|^{2}=\frac{c_{11} \mp \sqrt{\left|c_{11}\right|^{2}-\left|p_{11}\right|^{2}}}{2}
$$

From (5), it is easy to prove that $|A|^{2}$ is always larger than $|B|^{2}$, and we thus arrive at

$$
\begin{aligned}
|A|^{2} & =\frac{c_{11}+\sqrt{\left|c_{11}\right|^{2}-\left|p_{11}\right|^{2}}}{2} \\
|B|^{2} & =\frac{c_{11}-\sqrt{\left|c_{11}\right|^{2}-\left|p_{11}\right|^{2}}}{2} \\
r & =\frac{c_{11}-\sqrt{\left|c_{11}\right|^{2}-\left|p_{11}\right|^{2}}}{c_{11}+\sqrt{\left|c_{11}\right|^{2}-\left|p_{11}\right|^{2}}}
\end{aligned}
$$

\section{IMPLEMENTATION ISSUES OF THE PROPOSED AI-MVDR ALGORITHM}

Some implementation issues of the proposed AI-MVDR algorithm are next discussed, illustrating that it operates robustly for real-world measurements.

\section{A. Empirical Estimation of the Covariance and Pseudocovari- ance Matrix and Matrix Inversion}

Theoretically, the proposed algorithm converges to the actual system frequency; however, in the real world, the exact calculation of the gradient is not possible. It is calculated from the estimated covariance and pseudocovariance matrices, based upon the data matrix $\mathbf{V}(k)$, defined as [23]

$$
\begin{aligned}
& \mathbf{V}(k)=\left[\begin{array}{llll}
\mathbf{v}(k) & \mathbf{v}(k-1) & \ldots & \mathbf{v}(k-M+1)
\end{array}\right] \\
& =\left[\begin{array}{ccc}
v(k) & \ldots & v(k-M+1) \\
\vdots & \ddots & \vdots \\
v(n-K+1) & \ldots & v(n-M-K+2)]
\end{array}\right]
\end{aligned}
$$

where $M$ is the number of observations. The covariance $\mathrm{C}(k)$ and pseudocovariance matrix $\mathrm{P}(k)$, can be recursively estimated as

$$
\begin{aligned}
\mathrm{C}(k) & =\mathbf{V}(k) \mathbf{V}^{H}(k) \\
& =\mathrm{C}(k-1)-\frac{\mathbf{v}(k-M) \mathbf{v}^{H}(k-M)}{M}+\frac{\mathbf{v}(k) \mathbf{v}^{H}(k)}{M} \\
\mathrm{P}(k) & =\mathbf{V}(k) \mathbf{V}^{T}(k) \\
& =\mathrm{P}(k-1)-\frac{\mathbf{v}(k-M) \mathbf{v}^{T}(k-M)}{M}+\frac{\mathbf{v}(k) \mathbf{v}^{T}(k)}{M}
\end{aligned}
$$

while the inverse covariance matrix $\mathrm{C}^{-1}(k)$ can be estimated using the matrix inversion lemma [26] as:

$$
\begin{aligned}
\tilde{\mathrm{C}}^{-1}(k) & =\mathrm{C}^{-1}(k-1) \\
& +\frac{\mathrm{C}^{-1}(k-1) \mathbf{v}(k-M) \mathbf{v}^{H}(k-M) \mathbf{C}^{-1}(k-1)}{M-\mathbf{v}^{H}(k-M) \mathbf{C}^{-1}(k-1) \mathbf{v}(k-M)} \\
\mathrm{C}^{-1}(k) & =\tilde{\mathrm{C}}^{-1}(k)-\frac{\tilde{\mathrm{C}}^{-1}(k) \mathbf{v}(k) \mathbf{v}^{H}(k) \tilde{\mathbf{C}}^{-1}(k)}{M+\mathbf{v}^{H}(k) \tilde{\mathrm{C}}^{-1}(k) \mathbf{v}(k)}
\end{aligned}
$$

\section{B. Stability Analysis of the AI-MVDR Algorithm}

The recursion in (26) is based on the LMS stochastic gradient scheme, where the step-size $\mu$ plays a crucial role in the stability of such an estimator. To ensure satisfactory performance of the proposed algorithm, its theoretical stability with respect to $\mu$ is next analyzed.

Note that theoretically, $c_{11}=|A|^{2}+|B|^{2}=\sigma_{v}^{2}$ and $p_{11}=$ $2 A B=\rho_{v}^{2}$, hence (26) can be expressed as

$$
\begin{aligned}
\omega(k+1) & =\omega(k)+\mu \frac{2 \sigma_{v}^{2} \sqrt{\sigma_{v}^{4}-\left|\rho_{v}^{2}\right|^{2}}}{\sigma_{v}^{2}+\sqrt{\sigma_{v}^{4}-\left|\rho_{v}^{2}\right|^{2}}}\left(\sin \left(\omega_{\mathrm{o}}\right) \cos (\omega(k))\right. \\
& \left.-\cos \left(\omega_{0}\right) \sin (\omega(k))\right) \\
& =\omega(k)+\mu \frac{2 \sigma_{v}^{2} \sqrt{\sigma_{v}^{4}-\left|\rho_{v}^{2}\right|^{2}}}{\sigma_{v}^{2}+\sqrt{\sigma_{v}^{4}-\left|\rho_{v}^{2}\right|^{2}}} \sin \left(\omega_{0}-\omega(k)\right)
\end{aligned}
$$

Define the frequency estimation error as $\tilde{\omega}(k)=\omega_{0}-\omega(k)$, to have

$$
\tilde{\omega}(k+1)=\tilde{\omega}(k)-\mu \frac{2 \sigma_{v}^{2} \sqrt{\sigma_{v}^{4}-\left|\rho_{v}^{2}\right|^{2}}}{\sigma_{v}^{2}+\sqrt{\sigma_{v}^{4}-\left|\rho_{v}^{2}\right|^{2}}} \sin (\tilde{\omega}(k))
$$

Using the first term of the Taylor series expansion of $\sin (\tilde{\omega}(k))$, that is, $\tilde{\omega}(k)$, the condition for convergence in the mean, so that $|\tilde{\omega}(k+1)|<|\tilde{\omega}(k)|$ as $k \rightarrow \infty$, becomes

$$
-1<1-\mu \frac{2 \sigma_{v}^{2} \sqrt{\sigma_{v}^{4}-\left|\rho_{v}^{2}\right|^{2}}}{\sigma_{v}^{2}+\sqrt{\sigma_{v}^{4}-\left|\rho_{v}^{2}\right|^{2}}}<1
$$


TABLE I

THE IMPLEMENTATION OF THE PROPOSED AI-MVDR ALGORITHM

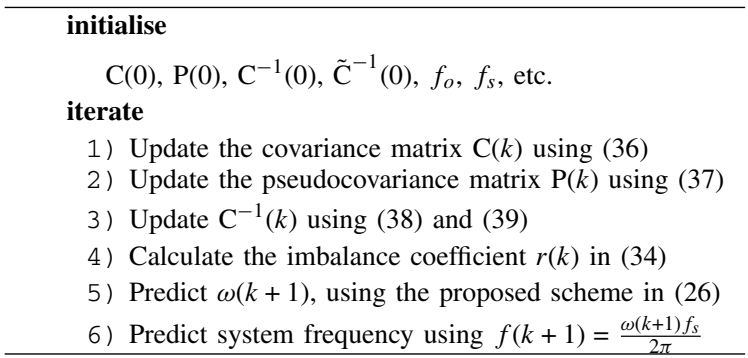

TABLE II

THE COMPUTATIONAL COMPLEXITY OF EACH STEP REQUiRed By THE AI-MVDR ALGORITHM PER ITERATION

\begin{tabular}{|c|c|c|}
\hline Steps & No. of real multiplications & No. of real additions \\
\hline 1 & $10 K^{2}$ & $6 K^{2}$ \\
\hline 2 & $12 K^{2}$ & $8 K^{2}$ \\
\hline 3 & $2 K^{3}+14 K^{2}$ & $2 K^{3}+12 K^{2}-8 K+2$ \\
\hline $4 / 5 / 6$ & $4 / 5 / 1$ & $4 / 4 / 0$ \\
\hline Total & $2 K^{3}+36 K^{2}+10$ & $2 K^{3}+26 K^{2}-8 K+10$ \\
\hline
\end{tabular}

thus imposing a bound on the step-size in the form

$$
0<\mu<\frac{\sigma_{v}^{2}+\sqrt{\sigma_{v}^{4}-\left|\rho_{v}^{2}\right|^{2}}}{\sigma_{v}^{2} \sqrt{\sigma_{v}^{4}-\left|\rho_{v}^{2}\right|^{2}}}
$$

It is also possible to relate the bound on the step-size $\mu$ in (43) to the amplitudes of the phase voltages, $V_{a}, V_{b}$, and $V_{c}$. Since $\sigma_{v}^{2}=|A|^{2}+|B|^{2}$ and $\rho_{v}^{2}=2 A B$, we obtain

$$
0<\mu<\frac{2|A|^{2}}{|A|^{4}-|B|^{4}}
$$

since from (5), $A$ and $B$ can be related to $V_{a}, V_{b}$, and $V_{c}$.

\section{The Implementation and Computational Complexity of AI- $M V D R$}

The steps, required for the implementation of the proposed AI-MVDR algorithm, are summarized in Table I. At each time instant $k$, the implementation of the original I-MVDR algorithm proposed in [1] requires Steps 3, 5, and 6, as it implicitly assumes that the imbalance coefficient $r(k)=0$. To compare the computational complexities of both algorithms, we here analyze the numbers of multiplications and additions required in each implementation step. Since in some steps realvalued operations are involved, the computation complexities are measured in terms of the real-valued multiplications and additions, ${ }^{3}$ and are summarized in Table II. Although the proposed algorithm requires additional Steps 1,2 , and 4 to calculate the update of the imbalance coefficient $r(k)$ as compared with the original algorithm, their computational complexities are comparable, $\mathcal{O}\left(K^{3}\right)$, as the calculation is dominated by the matrix inversion operation.

\footnotetext{
${ }^{3}$ Note that one complex multiplication requires four real multiplications and two real additions, while one complex addition requires two real additions. We here consider only the compulsory operations, as the constants, such as $\frac{1}{M}$ in Step 1 and $\frac{f_{s}}{2 \pi}$ in Step 6, can be predefined in the initialization stage.
}

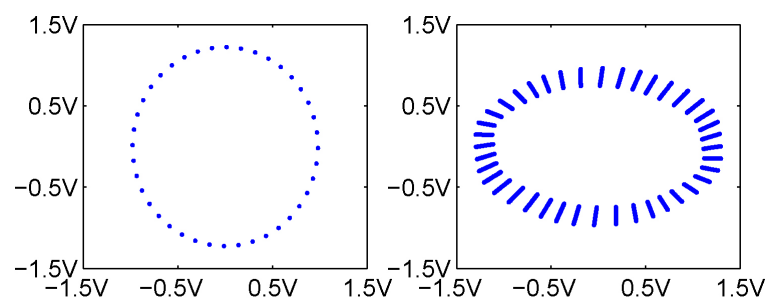

(a)

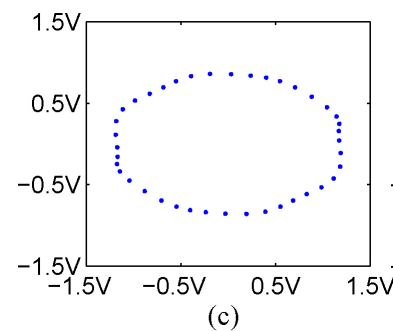

(c) (b)

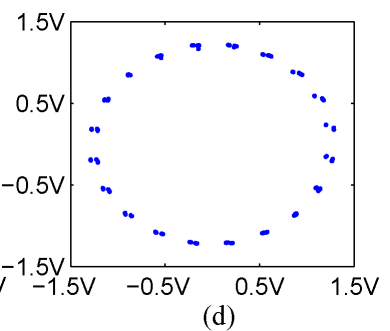

Fig. 1. Geometric view of the noncircularity of the complex-valued system voltage $v(k)$ via the 'real-imaginary' scatter plots. The horizontal axis represents the voltage magnitude of the real part of $v(k)$, whereas the vertical axis represents that of the imaginary part of $v(k)$. (a) Type B sag. (b) Type C sag, together with amplitude modulation and noise. (c) Type C sag, together with higher-order harmonics. (d) A real-world unbalanced power system. The complex-valued voltage $v(k)$ rotates anticlockwise as the time evolves.

\section{Simulations}

We considered frequency estimation under synthetic unbalanced voltage sag conditions and for a real world unbalanced power system. A voltage sag is referred to as a short-duration (up to a few seconds) reduction in voltage magnitude, whereby the three phase angles can also deviate from their nominal values. Voltage sags are mainly triggered by a short-term increase in load current, that may occur due to motor starting, transformer inrush, short circuits, or fast reclosing of circuit breakers [27]. Despite their short duration, such events can cause serious problems for a wide range of equipment and also cause difficulties in standard phase angle calculation, together with oscillatory estimation artifacts at twice the system frequency; this affects conventional PLL and strictly linear adaptive filters [16], [28]. It is worth mentioning that a system unbalance usually refers to steady state phenomena, including unequal voltage magnitudes at the fundamental system frequency (under-voltages and over-voltages), fundamental phase angle deviations, and unequal levels of harmonic distortion between the phases over a whole electrical grid for delivering electricity from suppliers to consumers, including power stations, transmission lines, transformers, power loads in the lower voltage consumption ends [29]. Voltage sags usually refer to unbalanced events happening at the lower voltage levels [27]. However, due to the well-defined mathematical modeling of voltage sags, for the illustration purpose, voltage sags were used in the synthetic simulation part related to the unbalanced power systems.

To quantify the characteristics of voltage sags, we here follow the phasor representation used in [27], and select two typical sags, known as Type B and Type C sags, for the illustration purpose. Type B sags result from a single-phase fault, causing one-phase voltage to drop while the two others 


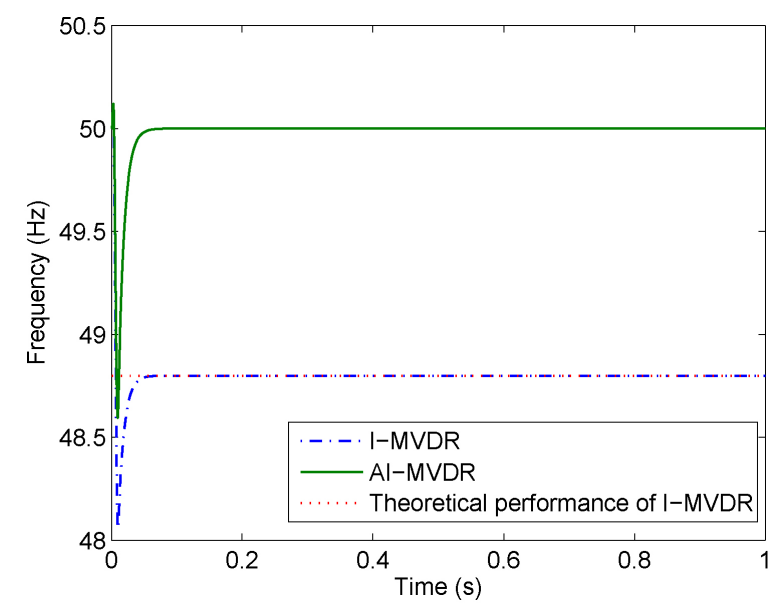

Fig. 2. Frequency estimation under an unbalanced Type B voltage sag condition. The initialized frequency of both algorithms was set to $50.1 \mathrm{~Hz}$, with the true system frequency at $50 \mathrm{~Hz}$.

remain unchanged. For a single fault happening on phase $v_{a}$

$$
\mathrm{V}_{a}=\gamma, \mathrm{V}_{b}=-\frac{1}{2}-\frac{J \sqrt{3}}{2}, \text { and } \mathrm{V}_{c}=-\frac{1}{2}+\frac{J \sqrt{3}}{2}
$$

whereas in a Type $\mathrm{C}$ voltage sag, two-phase voltages (assuming the phases $v_{b}$ and $v_{c}$ ) move toward each other, whereas the third phase remains unchanged, that is

$$
\mathrm{V}_{a}=1, \mathrm{~V}_{b}=-\frac{1}{2}-\frac{J \sqrt{3} \gamma}{2} \text {, and } \mathrm{V}_{c}=-\frac{1}{2}+\frac{J \sqrt{3} \gamma}{2}
$$

To illustrate the suitability of proposed AI-MVDR algorithm based on (26), a comparative performance analysis was performed against the original I-MVDR and the recently developed adaptive unbiased ACLMS algorithm [16], which requires 22 real multiplications and 18 real additions per iteration. Simulations were conducted in the MATLAB programming environment at a sampling frequency of $2000 \mathrm{~Hz}$. The observation length was set to $M=20$, and was used to estimate both the covariance and pseudocovariance matrix. In a power system with system frequency fixed at $50 \mathrm{~Hz}, 20$ observations sampled at $2000 \mathrm{~Hz}$ correspond to $10 \mathrm{~ms}$, which is a half-cycle of the $50 \mathrm{~Hz}$ voltage signal.

For the voltage sag modeling, we set the characteristic voltage to $\gamma=0.7$. The degree of noncircularity in unbalanced conditions was quantified using the noncircularity index $\eta$ [20]

$$
\eta=\frac{\left|\rho_{v}^{2}\right|}{\sigma_{v}^{2}}
$$

The values of the noncircularity index $\eta \in[0,1)$, with $\eta=$ 0 indicating that the complex-valued system voltage $v(k)$ is perfectly circular (balanced conditions), otherwise indicating a second-order noncircular $v(k)$ (unbalanced conditions).

\section{A. Synthetic Benchmark Cases}

In the first set of simulations, the simulated power system experienced a single-phase fault caused by a Type B voltage sag. The noncircularity index of the complex-valued system voltage $v(k)$ obtained by the $\alpha \beta$ transform of the three-phase voltage was $\eta=0.2194$. A geometric view of the distribution

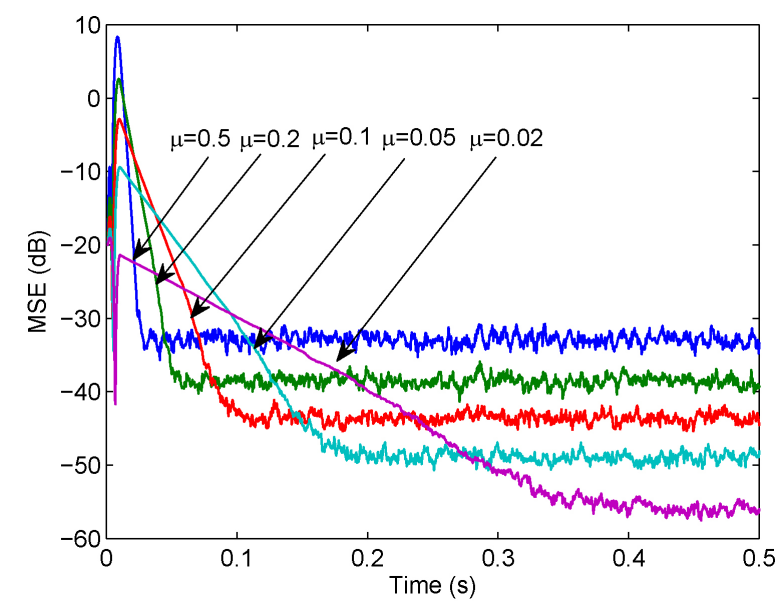

Fig. 3. Frequency estimation performance of the proposed AI-MVDR algorithm for a range of step sizes $\mu$, under a noisy unbalanced Type B voltage sag condition with $\mathrm{SNR}=40 \mathrm{~dB}$. The initialized frequency was set to be 50.1 $\mathrm{Hz}$, with the true system frequency at $50 \mathrm{~Hz}$.

via 'real-imaginary' plot is shown in Fig. 1(a), which illustrates the noncircular nature of the distribution of $v(k)$. This, in turn, indicates that the pseudocovariance matrix of $v(k)$ is not zero, and therefore the system imbalance coefficient $r(k)$ should be considered within the proposed AIMVDR iterative frequency estimation scheme, to compensate for the effect of system imbalance on frequency estimation (under-estimation). The frequency estimation using both I-MVDR and AI-MVDR algorithms with $\mu=0.2$ on the unbalanced power system is illustrated in Fig. 2. The proposed AI-MVDR algorithm was able to accurately estimate the true system frequency, whereas in the steady state the I-MVDR algorithm gave biased estimation, which conforms with theoretical performance analysis in (25).

In the next stage, the statistical performances of all the considered algorithms were tested in a noisy environment, based on the estimation bias and variance analysis. To that end, we first investigated the role of the step size $\mu$ in the MVDR spectrum-based algorithms. Note that in (9), the MVDR algorithms employ a LMS-type stochastic gradient approach to recursively minimize the convex cost function in (8). The role of the step size $\mu$ in stochastic gradient approaches is well studied in adaptive filters literature, as $\mu$ controls the tradeoff between the convergence speed and steady state estimation accuracy [20], [23]. A smaller step size leads to better steadystate performance and slower convergence, whereas a higher step-size results in faster convergence but larger steady-state estimation error. The adaptation and tracking performance of the MVDR spectrum-based algorithm is affected by the choice of $\mu$ in the same way. Fig. 3 illustrates the frequency estimation performance (in terms of the mean square error (MSE) in $\mathrm{dB}$ ) of the proposed AI-MVDR algorithm for a range of step sizes $\mu$, under a noisy unbalanced Type B voltage sag condition with SNR $=40 \mathrm{~dB}$. As expected, a smaller step size enables the proposed algorithm to track the system frequency more accurately (lower MSE) but at a cost of slower initial convergence. Ideally, we desire an algorithm for which the speed of convergence is fast and the steady-state estimation 


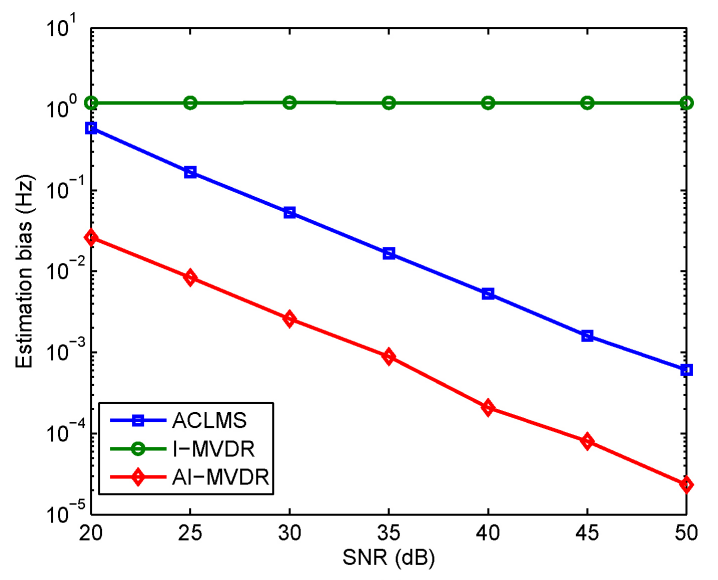

(a)

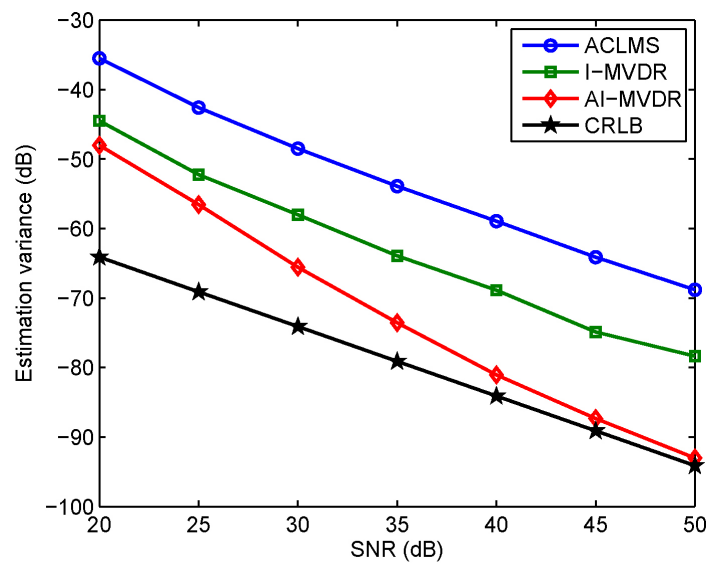

(b)

Fig. 4. Bias and variance analysis against the theoretical Cramer-Rao lower bound (CRLB) at different SNRs from 20 to 50 dB, obtained by averaging 1000 independent trials. (a) Estimation bias. (b) Estimation variance.

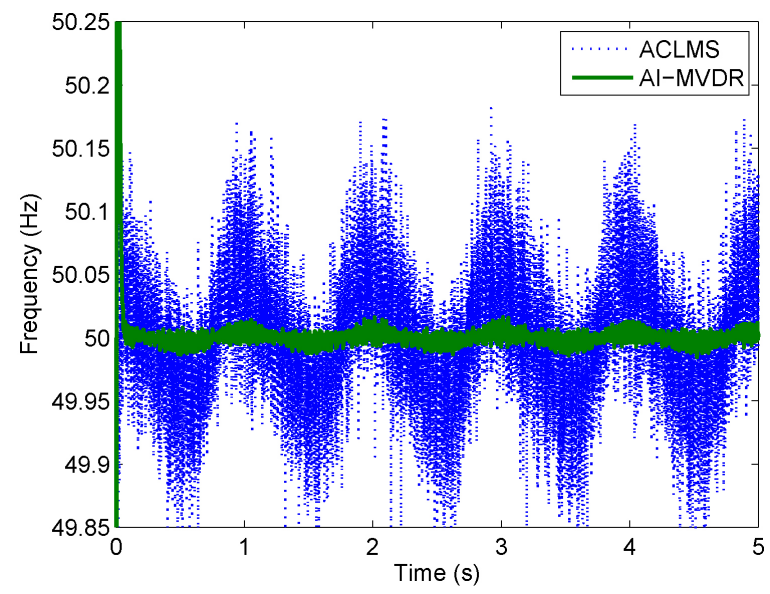

Fig. 5. Frequency estimation by ACLMS and AI-MVDR algorithms under Type $\mathrm{C}$ unbalanced voltage sag, together with voltage amplitude modulation.

error is small; this can be achieved by on-line adjusting the step size according to the input dynamics. We refer to [30], [31] for more detail.

We next performed the variance analysis by assessing the proposed algorithm against the theoretical CRLB, which characterizes the variance of an unbiased optimal frequency estimator based on the model in (4), contaminated by zero mean complex-valued doubly white circular Gaussian noise with variance $\sigma_{n}^{2}$ (Appendix A for the derivation). Fig. 4 illustrates the statistical bias and variance performance of all the considered algorithms, when applied to frequency estimation in an unbalanced system and against different levels of noise, where an optimal step size $\mu=0.0005$ was used in the MVDR spectrum-based algorithms. The results were obtained by averaging 1000 independent trials. The bias analysis, shown in Fig. 4(a), illustrates the unbiased property of the augmented ACLMS and the proposed AI-MVDR estimators that can be observed in the high SNR region, whereas due to the submodeling, I-MVDR always produced a biased estimate, which supports the theoretical analysis in (25). Also, observe that the bias error of I-MVDR algorithm was almost unaffected

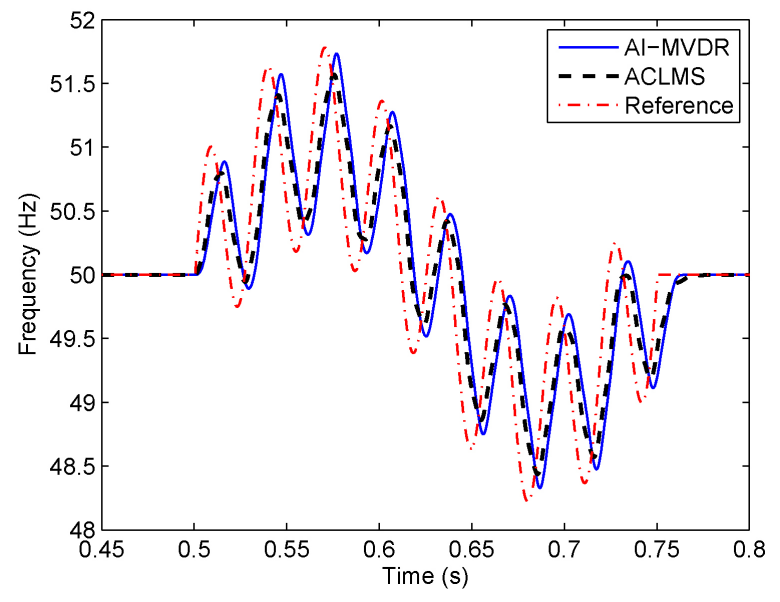

Fig. 6. Frequency estimation by ACLMS and AI-MVDR algorithms under Type $\mathrm{C}$ unbalanced voltage sag, together with frequency modulation.

by the level of noise. This can be explained by the fact that, compared with noise, the unavoidable theoretical bias resulting from the system imbalance within the gradient update, as shown in (25), was a major part of the estimation bias. In the variance analysis, as shown in Fig. 4(b), the AI-MVDR algorithm achieved the best performance among the algorithms considered, approaching the theoretical CRLB at high SNR.

In the next set of simulations, the performance under a more complex Type $\mathrm{C}$ voltage sag condition was assessed. This unbalanced power system first experienced a slow voltage amplitude modulation (AM) at $1 \mathrm{~Hz}$, where $V_{a}(k)=1+$ $0.05 \sin (2 \pi k \Delta \mathrm{T}), V_{b}(k)=1+0.1 \sin (2 \pi k \Delta \mathrm{T})$, and $V_{c}(k)=1+$ $0.15 \sin (2 \pi k \Delta \mathrm{T})$. Circular noise was added next, at $\mathrm{SNR}=50$ $\mathrm{dB}$, so that the resulting $v(k)$ was noncircular with a degree of noncircularity $\eta=0.34$, as shown in Fig. 1(b). Fig. 5 shows the tracking performance of the proposed AI-MVDR algorithm, with $\mu=0.2$, illustrating a very small estimation error with a maximum of $0.01 \mathrm{~Hz}$ frequency deviation, whereas the ACLMS suffered from large oscillations.

Next, frequency tracking ability of the proposed algorithm was considered. The unbalanced power system experienced a 


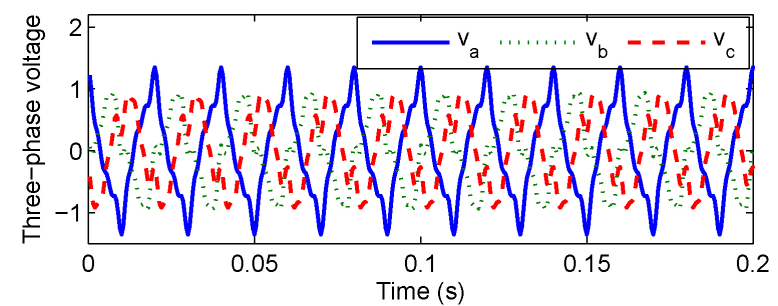

(a)

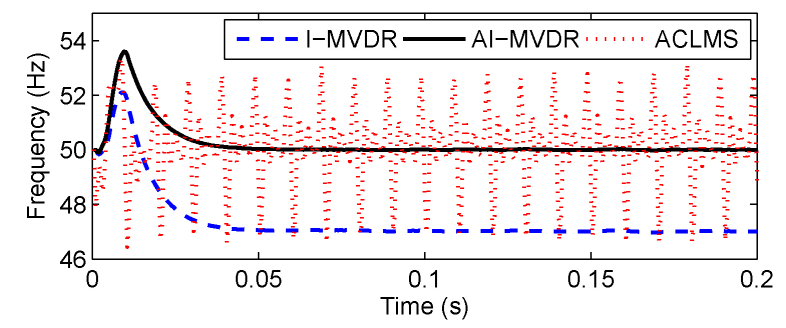

(b)

Fig. 7. Frequency estimation by all the algorithms under Type $\mathrm{C}$ unbalanced voltage sag, together with higher-order harmonics. (a) The waveforms of the three-phase voltage, which were distorted by $20 \% 3 \mathrm{rd}, 10 \%$ th, and $10 \%$ th harmonics. (b) Estimation performance.

combined sinusoid variations, described by

$$
f(t)=\left\{\begin{array}{l}
50+\sin (8 \pi(t-0.5))+ \\
0.8 \sin (64 \pi(t-0.5)), 0.5 \mathrm{~s} \leq t<0.75 \mathrm{~s} \\
50
\end{array}\right.
$$

elsewhere

Fig. 6 illustrates the frequency tracking performances of the AI-MVDR and ACLMS algorithms. Although both algorithms had similar response times, the proposed AI-MVDR algorithm (with $\mu=0.2$ ) tracked the frequency dynamics more accurately.

Next, to access the impact of higher-order harmonics on the performance of all the frequency estimators, the unbalanced system under Type C voltage sag was distorted by $20 \%$ $3 \mathrm{rd}, 10 \% 5 \mathrm{th}$, and $10 \%$ 7th harmonics of the fundamental frequency. The waveforms of this highly distorted threephase voltage are shown in Fig. 7(a), and their noncircular distributions in Fig. 1(c). From Fig. 7(b), observe that the frequency estimated by the ACLMS algorithm was subject to an oscillatory steady-state, whereas the proposed AI-MVDR algorithm was immune to higher-order harmonics distortions. This robustness is inherited from MVDR spectrum estimation, which together with the use of the augmented second-order statistics ensures excellent performance with a negligible bias at $0.0056 \mathrm{~Hz}$.

\section{B. Real World Case Study}

In the last set of simulations, a real-world power system was considered. The three-phase voltage was recorded at a 110/20/10 kV transformer station. The REL 531 numerical line distant protection terminal, produced by ABB Ltd., was installed in the station and was used to monitor changes in the three "phase-ground" voltages. The measured three "phaseground" voltages with a system frequency around $50 \mathrm{~Hz}$ were sampled at $1 \mathrm{kHz}$ and were normalized with respect to their normal peak voltage values. As shown in Fig. 8(a), phase $v_{b}$

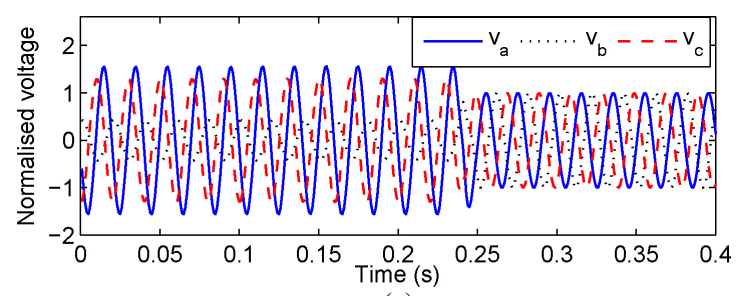

(a)

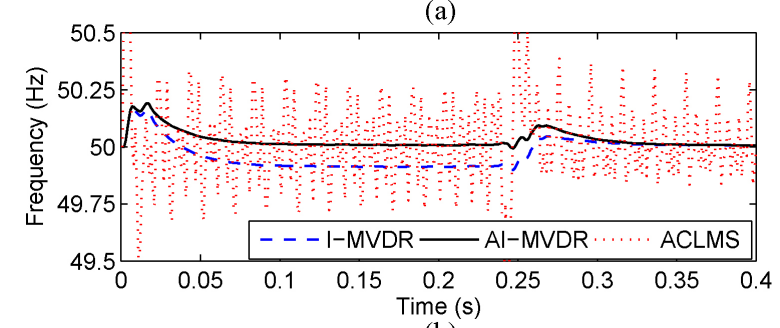

(b)

Fig. 8. Frequency estimation by all the algorithms for the real-world unbalanced three-phase voltage. (a) The waveforms of the three-phase voltage. (b) Frequency estimation for all algorithms.

first experienced an earth fault, causing a $56.5 \%$ voltage drop, and $55.3 \%$ and $29.5 \%$ voltage swells in phases $v_{a}$ and $v_{c}$ respectively, giving a degree of noncircularity of $\eta=0.0636$, with the noncircularity diagram of the system voltage $\mathrm{v}(\mathrm{k})$ shown in Fig. 1(d). At around $0.24 \mathrm{~s}$, the unbalanced voltage recovered to its nominal value. The frequency tracking capabilities for all the algorithms are shown in Fig. 8(b), and a system frequency at $50.008 \mathrm{~Hz}$ was suggested by the proposed AI-MVDR algorithm after its convergence. Conforming with the analysis in (25), the I-MVDR algorithm failed to deal with the unbalanced condition, giving biased estimation. When the system became balanced, both the proposed AI-MVDR and original I-MVDR algorithms converged. Compared with the unbiased adaptive ACLMS algorithm, the AI-MVDR achieved enhanced frequency estimation with no obvious oscillations in the steady state, and a smoother transient performance.

\section{CONCLUSION}

We introduced a robust AI-MVDR technique for online estimation of the fundamental frequency of unbalanced threephase power systems. This was achieved by combining a recently proposed iterative frequency estimation method based on the MVDR spectrum, and the full (augmented) secondorder statistics within the complex-valued system voltage. In this way, the proposed method inherits the advantages of the original approach, such as high estimation accuracy and fast convergence, and owing to the use of augmented complex statistics, it was a perfect match for frequency estimation in unbalanced system conditions, where the original approach suffered from unavoidable estimation bias. The AI-MVDR was more robust to noise, higher-order harmonics, and exhibited enhanced frequency tracking, compared to the recently introduced unbiased widely linear ACLMS estimation algorithm, as illustrated over a range of simulations. 


\section{APPENDIX A. THE VECTOR CRAMER-RAO LOWER BOUND FOR FREQUENCY ESTIMATION IN UNBALANCED SYSTEMS}

For a set of unknown parameters, $\boldsymbol{\theta}=\left[\theta_{1}, \ldots, \theta_{i}, \ldots, \theta_{L}\right]^{T}$, a joint probability density function (PDF) is $p(\mathbf{v} ; \boldsymbol{\theta})$, where $\mathbf{v}$ is a vector of observations. Then, according to the CRLB, the variance of any unbiased estimator $\hat{\theta}_{i}$ must satisfy [32], [33]

$$
\operatorname{var}\left(\hat{\theta}_{i}\right) \geq \mathbf{I}_{i i}^{-1}(\boldsymbol{\theta}), \quad i=1, \ldots, L
$$

where $\mathbf{I}(\boldsymbol{\theta})$ is the $L \times L$ Fisher information matrix, defined as

$$
\mathbf{I}_{i j}(\boldsymbol{\theta})=-E\left[\frac{\partial^{2} \ln (p(\mathbf{v} ; \boldsymbol{\theta}))}{\partial \theta_{i} \partial \theta_{j}}\right] \quad i, j=1, \ldots, L
$$

while $\mathbf{I}_{i i}^{-1}(\boldsymbol{\theta})$ is the $[i, i]$ element of the inverse of the Fisher information matrix. To evaluate (50), the true value of the unknown vector parameter $\boldsymbol{\theta}$ is used.

The complex-valued voltage $v(k)$ obtained from noisy unbalanced three-phase power systems obeys a widely linear model $v(k)=A e^{J(2 \pi f k \Delta T+\phi)}+B e^{-J(2 \pi f k \Delta T+\phi)}+n(k)$, where $n(k)$ is a complex-valued doubly white Gaussian noise $\sim \mathcal{N}\left(0, \sigma_{n}^{2}\right)$, where $n=n_{r}+\jmath n_{i}, \sigma_{n_{r}}^{2}=\sigma_{n_{i}}^{2}=\frac{\sigma_{n}^{2}}{2}$, and ${ }^{4} n_{r} \perp n_{i}$. The real and imaginary components, $v_{r}(k)$ and $v_{i}(k)$, are then

$$
\begin{aligned}
v(k) & =\underbrace{A \cos (2 \pi f k \Delta \mathrm{T}+\phi)+B_{r} \cos (2 \pi f k \Delta \mathrm{T}+\phi)+B_{i} \sin (2 \pi f k \Delta \mathrm{T}+\phi)+n_{r}(k)}_{v_{r}(k)} \\
& +\underbrace{\left(A \sin (2 \pi f k \Delta \mathrm{T}+\phi)+B_{i} \cos (2 \pi f k \Delta \mathrm{T}+\phi)-B_{r} \sin (2 \pi f k \Delta \mathrm{T}+\phi)+n_{i}(k)\right)}_{v_{i}(k)}
\end{aligned}
$$

while $B_{r}$ and $B_{i}$ are the real and imaginary parts of $B$.

When parameters in (51) are unknown, the vector parameter $\boldsymbol{\theta}=\left[f, A, B_{r}, B_{i}, \phi\right]^{T}$, and the likelihood function [33]

$$
\ln (p(\boldsymbol{v} ; \boldsymbol{\theta}))=-K \ln \left(\pi \sigma_{n}^{2}\right)-\frac{1}{\sigma_{n}^{2}} \sum_{k=1}^{K}\left(\left(v_{r}(k)-m_{r}(k)\right)^{2}+\left(v_{i}(k)-m_{i}(k)\right)^{2}\right)
$$

Thus, the Fisher information matrix is calculated from

$$
\mathrm{I}_{\theta_{i} \theta_{j}}=\frac{2}{\sigma_{n}^{2}} \sum_{k=1}^{K}\left(\frac{\partial m_{r}}{\partial \theta_{i}} \frac{\partial m_{r}}{\partial \theta_{j}}+\frac{\partial m_{i}}{\partial \theta_{i}} \frac{\partial m_{i}}{\partial \theta_{j}}\right)
$$

while for $\frac{\partial m_{r}(k)}{\partial \boldsymbol{\theta}}=\left[\frac{\partial m_{r}(k)}{\partial f}, \frac{\partial m_{r}(k)}{\partial A}, \frac{\partial m_{r}(k)}{\partial B_{r}}, \frac{\partial m_{r}(k)}{\partial B_{i}}, \frac{\partial m_{r}(k)}{\partial \phi}\right]^{T}$

$$
\frac{\partial m_{r}(k)}{\partial \boldsymbol{\theta}}=\left[\begin{array}{c}
2 \pi k \Delta \mathrm{T}\left(B_{i} \cos (2 \pi f k \Delta \mathrm{T}+\phi)-\left(A+B_{r}\right) \sin (2 \pi f k \Delta \mathrm{T}+\phi)\right) \\
\cos (2 \pi f k \Delta \mathrm{T}+\phi) \\
\cos (2 \pi f k \Delta \mathrm{T}+\phi) \\
\sin (2 \pi f k \Delta \mathrm{T}+\phi) \\
B_{i} \cos (2 \pi f k \Delta \mathrm{T}+\phi)-\left(A+B_{r}\right) \sin (2 \pi f k \Delta \mathrm{T}+\phi)
\end{array}\right]
$$

and for $\frac{\partial m_{i}(k)}{\partial \theta}=\left[\frac{\partial m_{i}(k)}{\partial f}, \frac{\partial m_{i}(k)}{\partial A}, \frac{\partial m_{i}(k)}{\partial B_{r}}, \frac{\partial m_{i}(k)}{\partial B_{i}}, \frac{\partial m_{i}(k)}{\partial \phi}\right]^{T}$

$$
\frac{\partial m_{i}(k)}{\partial \boldsymbol{\theta}}=\left[\begin{array}{c}
2 \pi k \Delta \mathrm{T}\left(\left(A-B_{r}\right) \cos (2 \pi f k \Delta \mathrm{T}+\phi)-B_{i} \sin (2 \pi f k \Delta \mathrm{T}+\phi)\right) \\
\sin (2 \pi f k \Delta \mathrm{T}+\phi) \\
-\sin (2 \pi f k \Delta \mathrm{T}+\phi) \\
\cos (2 \pi f k \Delta \mathrm{T}+\phi) \\
\left(A-B_{r}\right) \cos (2 \pi f k \Delta \mathrm{T}+\phi)-B_{i} \sin (2 \pi f k \Delta \mathrm{T}+\phi)
\end{array}\right]
$$

${ }^{4}$ The independence between $n_{r}$ and $n_{i}$ and their equal powers can be achieved by assuming the three-phase channels contaminated by independent and identically distributed zero-mean Gaussian noises with variance $\frac{\sigma_{n}^{2}}{2}$.
Therefore,

$$
\begin{aligned}
& \mathbf{I}_{11}=\frac{2}{\sigma_{n}^{2}} \sum_{k=1}^{K}(2 \pi k \Delta \mathrm{T})^{2}\left(A^{2}+B_{r}^{2}+B_{i}^{2}-2 A\left(B_{r} \cos (4 \pi f k \Delta \mathrm{T}+2 \phi)+B_{i} \sin (4 \pi f k \Delta \mathrm{T}+2 \phi)\right)\right) \\
& \mathbf{I}_{12}=\mathbf{I}_{21}=\frac{2}{\sigma_{n}^{2}} \sum_{k=1}^{K} 2 \pi k \Delta \mathrm{T}\left(B_{i} \cos (4 \pi f k \Delta \mathrm{T}+2 \phi)-B_{r} \sin (4 \pi f k \Delta \mathrm{T}+2 \phi)\right) \\
& \mathbf{I}_{13}=\mathbf{I}_{31}=\frac{2}{\sigma_{n}^{2}} \sum_{k=1}^{K} 2 \pi k \Delta \mathrm{T}\left(B_{i}-A \sin (4 \pi f k \Delta \mathrm{T}+2 \phi)\right) \\
& \mathbf{I}_{14}=\mathbf{I}_{41}=\frac{2}{\sigma_{n}^{2}} \sum_{k=1}^{K} 2 \pi k \Delta \mathrm{T}\left(A \cos (4 \pi f k \Delta \mathrm{T}+2 \phi)-B_{r}\right) \\
& \mathbf{I}_{15}=\mathbf{I}_{51}=\frac{2}{\sigma_{n}^{2}} \sum_{k=1}^{K} 2 \pi k \Delta \mathrm{T}\left(A^{2}+B_{r}^{2}+B_{i}^{2}-2 A\left(B_{r} \cos (4 \pi f k \Delta \mathrm{T}+2 \phi)+B_{i} \sin (4 \pi f k \Delta \mathrm{T}+2 \phi)\right)\right) \\
& \mathbf{I}_{22}=\mathbf{I}_{33}=\mathbf{I}_{44}=\frac{2 K}{\sigma_{n}^{2}}, \mathbf{I}_{34}=\mathbf{I}_{43}=0 \\
& \mathbf{I}_{23}=\mathbf{I}_{32}=\frac{2}{\sigma_{n}^{2}} \sum_{k=1}^{K} \cos (4 \pi f k \Delta \mathrm{T}+2 \phi), \mathbf{I}_{24}=\mathbf{I}_{42}=\frac{2}{\sigma_{n}^{2}} \sum_{k=1}^{K} \sin (4 \pi f k \Delta \mathrm{T}+2 \phi) \\
& \mathbf{I}_{25}=\mathbf{I}_{52}=\frac{2}{\sigma_{n}^{2}} \sum_{k=1}^{K}\left(B_{i} \cos (4 \pi f k \Delta \mathrm{T}+2 \phi)-B_{r} \sin (4 \pi f k \Delta \mathrm{T}+2 \phi)\right) \\
& \mathbf{I}_{35}=\mathbf{I}_{53}=\frac{2}{\sigma_{n}^{2}} \sum_{k=1}^{K}\left(-A \sin (4 \pi f k \Delta \mathrm{T}+2 \phi)+B_{i} \cos (4 \pi f k \Delta \mathrm{T}+2 \phi)\right) \\
& \mathbf{I}_{45}=\mathbf{I}_{54}=\frac{2}{\sigma_{n}^{2}} \sum_{k=1}^{K}\left(A \cos (4 \pi f k \Delta \mathrm{T}+2 \phi)-B_{r}\right) \\
& \mathbf{I}_{55}=\frac{2}{\sigma_{n}^{2}} \sum_{k=1}^{K}\left(A^{2}+B_{r}^{2}+B_{i}^{2}-2 A\left(B_{r} \cos (4 \pi f k \Delta \mathrm{T}+2 \phi)+B_{i} \sin (4 \pi f k \Delta \mathrm{T}+2 \phi)\right)\right)
\end{aligned}
$$

This Fisher information matrix also accounts for the complex exponential with the negative frequency to model the noncircularity of the unbalanced system voltage. From (50), a general CRLB for unbiased frequency estimator on unbalanced threephase power system can be obtained as

$$
\operatorname{var}(\hat{f}) \geq \mathbf{I}_{11}^{-1}
$$

where $\mathbf{I}_{11}^{-1}$ is the first element in the inverse of Fisher information matrix, which was evaluated empirically in Fig. 4(b).

\section{ACKNOWLEDGMENT}

We wish to thank Z. Blazic of Elektroprenos, BiH, for providing real-world data, fruitful discussions, and expert advice.

\section{REFERENCES}

[1] H.-J. Jeon and T.-G. Chang, "Iterative frequency estimation based on MVDR spectrum," IEEE Trans. Power Del., vol. 25, no. 2, pp. 621-630, Mar. 2010.

[2] M. S. Sachdev and M. M. Giray, "A least error squares technique for determining power system frequency," IEEE Trans. Power App. Syst., vol. PAS-104, no. 5, pp. 1025-1038, Feb. 1983.

[3] T. S. Sidhu, "Accurate measurement of power system frequency using a digital signal processing technique," IEEE Trans. Instrum. Meas. vol. 48, no. 3, pp. 75-81, Feb. 1999.

[4] G. P. Hancke, "The optimal frequency estimation of a noisy sinusoidal signal," IEEE Trans. Instrum. Meas., vol. 39, no. 6, pp. 843-846, Dec. 1990.

[5] S. K. Chung, "A phase tracking system for three phase utility interface inverters," IEEE Trans. Power Electron., vol. 15, no. 3, pp. 431-438, May 2000.

[6] A. K. Pradhan, A. Routray, and A. Basak, "Power system frequency estimation using least mean square technique," IEEE Trans. Power Del., vol. 20, no. 3, pp. 761-766, Jun. 2005.

[7] V. V. Terzija, "Improved recursive Newton-type algorithm for frequency and spectra estimation in power systems," IEEE Trans. Instrum. Meas., vol. 52, no. 5, pp. 1654-1659, Oct. 2003.

[8] P. K. Dash, A. K. Pradhan, and G. Panda, "Frequency estimation of distorted power system signals using extended complex Kalman filter," IEEE Trans. Power Del., vol. 14, no. 3, pp. 761-766, Jul. 1999. 
[9] M. Mojiri, D. Yazdani, and A. Bakhshai, "Robust adaptive frequency estimation of three-phase power system," IEEE Trans. Instrum. Meas., vol. 59, no. 7, pp. 1793-1802, Jul. 2010.

[10] M. Akke, "Frequency estimation by demodulation of two complex signals," IEEE Trans. Power Del., vol. 12, no. 1, pp. 157-163, Jan. 1997.

[11] R. O. Schmidt, "Multiple emitter location and signal parameter estimation," IEEE Trans. Antenn. Propagat., vol. 34, no. 3, pp. 276-280, Mar. 1986.

[12] I. Y. H. Gu and M. H. J. Bollen, "Estimating interharmonics by using sliding-window ESPRIT," IEEE Trans. Power Del., vol. 23, no. 1, pp. 13-23, Jan. 2008

[13] Electromagnetic Compatibility (EMC): Testing and Measurement Techniques-Power Quality Measurement Methods, IEC 61000-4-30, 2003.

[14] A. Ghosh and A. Joshi, "A new algorithm for the generation of reference voltages of a DVR using the method of instantaneous symmetrical components," IEEE Power Eng. Rev., vol. 22, no. 1, pp. 63-65, Jan. 2002

[15] M. Karimi-Ghartemani and M. R. Iravani, "A method for synchronization of power electronic converters in polluted and variable-frequency environments," IEEE Trans. Power Syst., vol. 19, no. 3, pp. 1263-1270, Aug. 2004.

[16] Y. Xia and D. P. Mandic, "Widely linear adaptive frequency estimation of unbalanced three-phase power system," IEEE Trans. Instrum. Meas., vol. 61, no. 1, pp. 74-83, Jan. 2012.

[17] Y. Xia, S. C. Douglas, and D. P. Mandic, "Adaptive frequency estimation in smart grid applications: Exploiting noncircularity and widely linear adaptive estimators," IEEE Signal Process. Mag., vol. 29, no. 5, pp. 44-54, Aug. 2012.

[18] B. Picinbono, "On circularity," IEEE Trans. Signal Process., vol. 42, no. 12 , pp. 3473-3482, Dec. 1994

[19] B. Picinbono and P. Chevalier, "Widely linear estimation with complex data," IEEE Trans. Signal Process., vol. 43, no. 8, pp. 2030-2033, Aug. 1995.

[20] D. P. Mandic and S. L. Goh, Complex Valued Nonlinear Adaptive Filters: Noncircularity, Widely Linear and Neural Models. New York, NY, USA Wiley, 2009.

[21] Y. Xia, B. Jelfs, M. M. V. Hulle, and J. C. Príncipe, "An augmented echo state network for nonlinear adaptive filtering of complex noncircular signals," IEEE Trans. Neural Netw., vol. 22, no. 1, pp. 74-83, Jan. 2011

[22] J. Capon, "High resolution frequency wave number spectrum analysis," Proc. IEEE, vol. 57, no. 8, pp. 1408-1418, Aug. 1969.

[23] S. Haykin, Adaptive Filter Theory. New Jersey, NJ, USA: Prentice-Hall, 2002.

[24] B. Widrow and S. D. Stearns, Adaptive Signal Processing. New Jersey, NJ, USA: Prentice-Hall, 1985.

[25] P. Chevalier and A. Blin, "Widely linear MVDR beamformers for the reception of an unknown signal corrupted by noncircular interferences," IEEE Trans. Signal Process., vol. 55, no. 11, pp. 2181-2186, Nov. 2007.

[26] G. H. Golub and C. F. V. Loan, Matrix Computations. Maryland, MD, USA: Johns Hopkins Univ. Press, 1996.

[27] M. H. J. Bollen, "Characterisation of voltage sags experienced by threephase adjustable-speed drives," IEEE Trans. Power Del., vol. 12, no. 4 , pp. 1666-1671, Oct. 1997.
[28] M. D. Kušljević, J. J. Tomić, and L. D. Jovanović, "Frequency estimation of three-phase power system using weighted-least-square algorithm and adaptive FIR filtering," IEEE Trans. Instrum. Meas., vol. 57, no. 10, pp. 322-329, Feb. 2010.

[29] A. von Jouanne and B. Banerjee, "Assessment of voltage unbalance," IEEE Trans. Power Del., vol. 14, no. 4, pp. 782-790, Oct. 2001.

[30] W. Ang and B. Farhang-Boroujeny, "A new class of gradient adaptive step-size LMS algorithms," IEEE Trans. Signal Process., vol. 49, no. 4, pp. 805-810, Apr. 2001.

[31] D. P. Mandic, "A generalized normalized gradient descent algorithm," IEEE Signal Process. Lett., vol. 11, no. 2, pp. 115-118, Feb. 2004.

[32] S. M. Kay, Fundamentals of Statistical Signal Processing: Estimation Theory. New Jersey, NJ, USA: Prentice Hall, 1993.

[33] D. C. Rife and R. R. Boorstyn, "Single-tone parameter estimation from discrete-time observations," IEEE Trans. Inform. Theory, vol. 20, no. 5, pp. 591-598, Sep. 1974

Yili Xia (M'11) received the B.Eng. degree in information engineering from Southeast University, Nanjing, China, in 2006, the M.Sc. (Distinction) degree in communications and signal processing from the Department of Electrical and Electronic Engineering, Imperial College London, London, U.K., in 2007, and the $\mathrm{Ph} . \mathrm{D}$. degree in adaptive signal processing from Imperial College, London, U.K., in 2011.

Since his graduation, he has been a Research Associate with Imperial College London, and is currently an Associate Professor with the School of Information and Engineering, Southeast University, Nanjing, China. His current research interests include complex-valued linear and nonlinear adaptive filters, complex-valued statistical analysis, and their applications on power systems.

Dr. Xia was a co-recipient of the Best Student Paper Award at the International Symposium on Neural Networks in 2010.

Danilo P. Mandic (M'99-SM'03-F'12) received the Ph.D. degree in nonlinear adaptive signal processing from Imperial College London, London, U.K., in 1999, where he is currently a Professor of signal processing.

He has been engaged in the area of nonlinear adaptive signal processing, multivariate data analysis, and nonlinear dynamics. He has been a Guest Professor at Katholieke Universiteit Leuven, Leuven, Belgium, the Tokyo University of Agriculture and Technology, Tokyo, Japan, and Westminster University, London, and a Frontier Researcher at RIKEN, Japan. His publication record includes two research monographs entitled, Recurrent Neural Networks for Prediction: Learning Algorithms, Architectures and Stability (1st ed., Aug. 2001) and Complex Valued Nonlinear Adaptive Filters: Noncircularity, Widely Linear and Neural Models (1st ed., Wiley, Apr. 2009), an edited book entitled, Signal Processing Techniques for Knowledge Extraction and Information Fusion (Springer, 2008) and more than 200 publications on signal and image processing.

Dr. Mandic has been a member of the IEEE Technical Committee on SIGNAL PROCESSING THEORY and METHODS, and an Associate Editor for the IEEE SIGNAL PROCESSING MAGAZINE, IEEE TRANSACTIONS on CIRCUITS and SYSTEMS II, IEEE TRANSACTIONS on SIGNAL PROCESSING, IEEE TRANSACTIONS on NEURAL NEETWORKS, and the INTERNATIONAL JOURNAL of MATHEMATICAL MODELLING and ALGORITHMS. He has produced award winning papers and products resulting from his collaboration with the industry. 